\title{
An in-silico analysis of the effect of heart position and orientation on the ECG morphology and vectorcardiogram parameters in patients with heart failure and intraventricular conduction defects
}

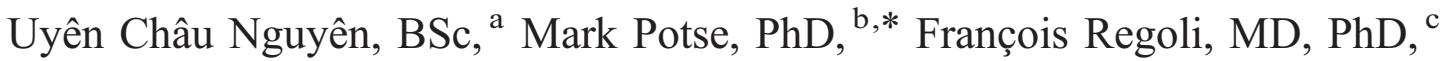 \\ Maria Luce Caputo, MD, ${ }^{\mathrm{c}}$ Giulio Conte, $\mathrm{MD}, \mathrm{PhD},{ }^{\mathrm{c}}$ Romina Murzilli, MD, ${ }^{\mathrm{c}}$ \\ Stefano Muzzarelli, MD, ${ }^{\mathrm{c}}$ Tiziano Moccetti, MD, ${ }^{\mathrm{c}}$ Enrico G. Caiani, PhD, ${ }^{\mathrm{d}}$ \\ Frits W. Prinzen, $\mathrm{PhD},{ }^{\mathrm{e}}$ Rolf Krause, $\mathrm{PhD},{ }^{\mathrm{b}}$ Angelo Auricchio, MD, $\mathrm{PhD}{ }^{\mathrm{b}, \mathrm{c}}$ \\ ${ }^{a}$ Faculty of Medicine, Maastricht University, Maastricht, the Netherlands \\ ${ }^{\mathrm{b}}$ Center for Computational Medicine in Cardiology, Institute of Computational Science, Università della Svizzera italiana, Lugano, Switzerland \\ c Division of Cardiology, Fondazione Cardiocentro Ticino, Lugano, Switzerland \\ d Dipartimento di Elettronica, Informazione e Bioingegneria, Politecnico di Milano, Milan, Italy \\ e Department of Physiology, Cardiovascular Research Institute Maastricht, Maastricht, the Netherlands
}

\begin{abstract}
Aim: The aim of this study was to investigate the influence of geometrical factors on the ECG morphology and vectorcardiogram (VCG) parameters.

Methods: Patient-tailored models based on five heart-failure patients with intraventricular conduction defects (IVCDs) were created. The heart was shifted up to $6 \mathrm{~cm}$ to the left, right, up, and down and rotated $\pm 30^{\circ}$ around the anteroposterior axis. Precordial electrodes were shifted $3 \mathrm{~cm}$ down.

Results: Geometry modifications strongly altered ECG notching/slurring and intrinsicoid deflection time. Maximum VCG parameter changes were small for QRS duration $(-6 \%$ to $+10 \%)$ and QRS-T angle $(-6 \%$ to $+3 \%)$, but considerable for QRS amplitude $(-36 \%$ to $+59 \%)$, QRS area $(-37 \%$ to $+42 \%)$, T-wave amplitude ( $-41 \%$ to $+36 \%)$, and T-wave area $(-42 \%$ to $+33 \%)$.

Conclusion: The position of the heart with respect to the electrodes is an important factor determining notching/slurring and voltage-dependent parameters and therefore must be considered for accurate diagnosis of IVCDs.
\end{abstract}

(C) 2015 Elsevier Inc. All rights reserved.

Keywords: $\quad$ Geometry; ECG morphology; VCG; Computer simulation

\section{Introduction}

Intraventricular conduction defects (IVCDs) like left bundle branch block (LBBB) on the electrocardiogram (ECG) are important predictors for response to cardiac resynchronization therapy (CRT) [1]. Therefore, accurate evaluation of the 12-lead ECG is important for the selection of patients for CRT. Several detailed ECG morphology criteria for the diagnosis of LBBB exist [2-4].

The ECG morphology is sensitive to geometrical factors such as heart-torso geometry, body position, respiration, and

* Corresponding author at: Center for Computational Medicine in Cardiology, Institute of Computational Science, Faculty of Informatics, Università della Svizzera italiana, Via Giuseppe Buffi 13, 6904 Lugano, Switzerland.

E-mail address: mark@potse.nl body habitus $[5,6]$. Moreover, ventricular enlargement, as noted in heart-failure (HF) patients, may rotate the heart around the anteroposterior axis to a more horizontal orientation [7]. All these factors may affect the interpretation of the ECG and the diagnosis of cardiac pathologies, and possibly influence indication to device therapy [8].

The aim of this study was to investigate the influence of geometrical factors on the 12-lead ECG signal. This was performed in-silico, using tailored models of patients with a wide range of $\mathrm{QRS}$ duration (QRSd) and QRS morphology. Geometry modifications were induced by shifting and rotating the heart and shifting the precordial electrodes. Alterations in the ECG signal were assessed morphologically, in the context of LBBB, and quantitatively, using parameters from the reconstructed vectorcardiogram (VCG). 


\section{Material and methods}

\section{Patient characteristics}

Five heart-failure (HF) patients (New York Heart Association class (NYHA) $\geq$ II) referred for CRT implantation and presenting with LBBB or aspecific IVCDs were studied. Data acquisition took place between April and July 2012 at Cardiocentro Ticino.

All patients underwent a standard 12-lead ECG, a cardiac magnetic resonance (CMR) scan, electroanatomical mapping, and a coronary angiography. The data collection approach has been described previously [9]. All diagnostic procedures were medically indicated. Written consent from the patients and approval of the institutional review board were obtained for the use of these data for research purposes.

\section{Segmentation and reconstruction of anatomical structures}

CMR data were obtained using a $3 \mathrm{~T}$ Siemens Magnetom Skyra scanner. These data were used to trace the contours of anatomical structures using custom software. The ventricular epicardium and endocardium were segmented semi-automatically from ECG-triggered mid-diastatic segmented steady-state free precession images with a slice thickness of 8 mm. The atria, pulmonary trunk, and aorta were manually segmented from a navigator-gated, ECG-triggered whole-heart angiography with a T1-weighted inversion-recovery echo-gradient sequence with a slice thickness of $0.9 \mathrm{~mm}$, and with inversion time (TI) adjusted using TI-scout images. The lungs and torso were segmented from a stack of ultra-fast T1-weighted gradient-echo images obtained after intravenous bolus injection of gadolinium (Gadobutrol, $0.2 \mathrm{mmol} / \mathrm{kg}$ body weight).

The segmentation data were used to create a surface mesh of the tissue boundaries using the Blender software (The Blender Foundation, Amsterdam, the Netherlands). Separate structures were linked to form a 3D model of the heart with its surrounding anatomy. From this model a computational mesh was formed. Mesh nodes were labeled tissue-specifically and fiber orientations were assigned to the ventricular nodes using a rule-based method [10]. To compare simulated and measured activation times, a set of catheter locations from the electroanatomical mapping system was aligned with the LV endocardium [9].

\section{Computer simulations}

Electrophysiological simulations were performed using propag-5 [10]. Computations were performed on a Cray XE6 supercomputer operated by the Swiss National Supercomputing Centre CSCS.

A ventricular model with a $0.2-\mathrm{mm}$ resolution and an inhomogeneous torso model with 1-mm resolution were used for the simulations. Propagating electrical activity was simulated based on ionic transmembrane currents according to a monodomain reaction-diffusion equation [11]. The Ten Tusscher-Noble-Noble-Panfilov membrane model for human ventricular myocytes was used to compute the ionic currents.

Computed transmembrane currents were injected at 1-ms intervals in the torso model and the bidomain equation was solved for the electrical potential throughout the torso, from which the 12-lead ECG was extracted [11]. At baseline, each model was tuned to match the simulated ECG with the measured ECG [9].

\section{Changes in heart position and orientation}

The heart was shifted with 1-cm steps up to $6 \mathrm{~cm}$ to the left and to the right along the $\mathrm{x}$-axis and up and down along the $\mathrm{z}$-axis as these shift magnitudes were used in previous studies [5,12]. The heart was rotated with $5^{\circ}$ steps up to $30^{\circ}$ around the y-axis (anteroposterior) to a more horizontal or vertical orientation. The rotation axis was placed between the base of the aorta and the pulmonary trunk. The precordial leads V1-V6 were shifted up to $3 \mathrm{~cm}$ down with $0.5-\mathrm{cm}$ steps as literature showed that overall $64 \%$ of precordial electrodes are placed within a radius of 1.25 inches [13]. Geometry modifications with their accompanying axes are illustrated in Fig. 1.

\section{Evaluation of ECG morphology}

The evaluation of the ECG morphology was limited to LBBB and IVCDs using the ECG parameters recommended by the European Society of Cardiology (ESC) [2], the American Heart Association (AHA) [3], and Strauss et al. [4] as represented in Table 1. Morphology parameters were evaluated individually and in the context of LBBB/non-LBBB diagnosis.

\section{Quantitative evaluation}

To quantify the differences between the ECG signals at baseline simulation and after geometry modifications, VCG parameters were used. The VCG was reconstructed from the 12-lead ECG with the Kors transformation matrix, as this method has been shown to resemble the Frank VCG the best [14].

The following parameters were assessed: QRSd [ms], QRS amplitude [mV], QRS area [mV·ms], QRS-T angle [ $\left.{ }^{\circ}\right], \mathrm{T}^{-w a v e}$ amplitude $[\mathrm{mV}]$, and T-wave area $[\mathrm{mV} \cdot \mathrm{ms}]$, as previous studies have demonstrated that these are predictors for CRT outcome and for sudden cardiac death [14-17].

The vector magnitude of the VCG $\left(\mathrm{V}_{\mathrm{VCG}}\right)[\mathrm{mV}]$ was computed from the three $\mathrm{VCG}$ leads $\mathrm{V}_{\mathrm{x}}, \mathrm{V}_{\mathrm{y}}$, and $\mathrm{V}_{\mathrm{z}}$ as: $V_{V C G}=\left(V_{x}^{2}+V_{y}^{2}+V_{z}^{2}\right)^{1 / 2}$

and was used to derive QRSd, QRS amplitude, and T-wave amplitude. The QRS end point was precisely defined using the local minimum around the J-point. QRS amplitude and T-wave amplitude were defined as the peak voltage in the QRS complex and T wave respectively. 

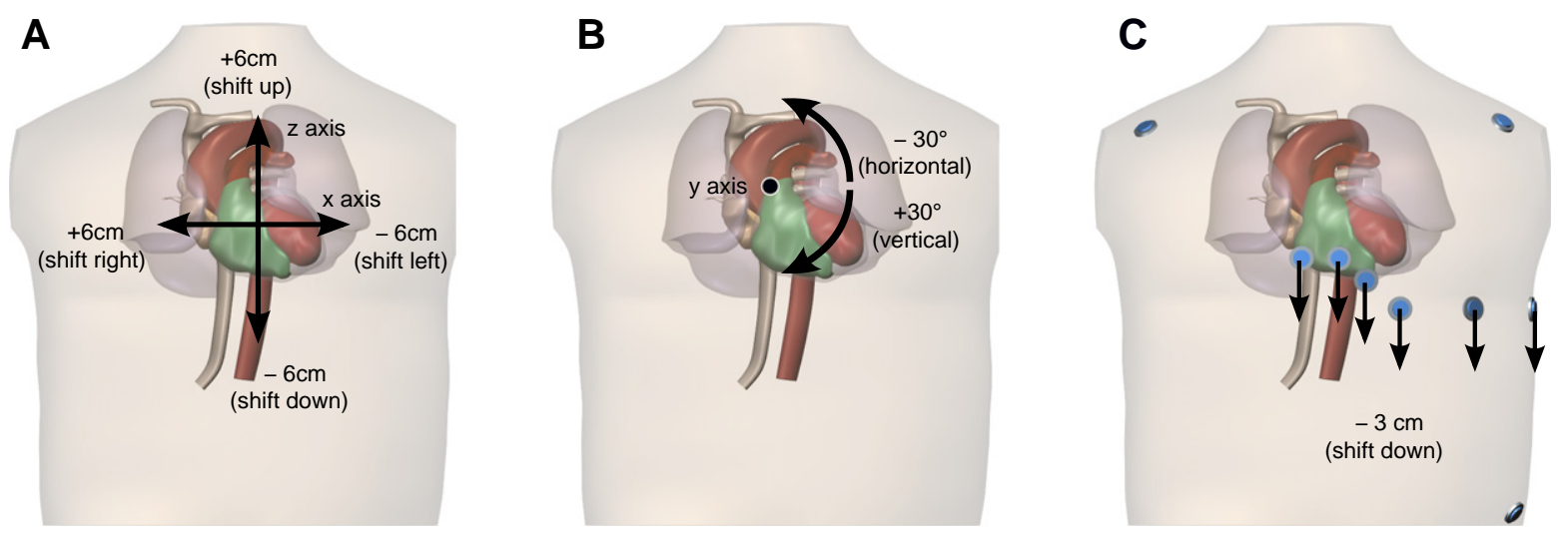

Fig. 1. Geometry modifications. A. The heart is translated up to $6 \mathrm{~cm}$ to the left, right, up, and down. B. The heart is rotated up to $30^{\circ}$ around the anteroposterior axis to a more horizontal and vertical orientation. C. The precordial electrodes V1-V6 are shifted up to $3 \mathrm{~cm}$ downward. Note the minus and plus signs for the corresponding directions.

The QRS area was calculated from the time-voltage area of the QRS complex in the $\mathrm{X}, \mathrm{Y}$, and Z leads $\left(A_{\mathrm{QRS}, x}, \mathrm{~A}_{\mathrm{QRS}, y}, \mathrm{~A}_{\mathrm{QRS}, z}\right)$ of the VCG $[15,16]$ :

$A_{\mathrm{QRS}}=\left(A_{\mathrm{QRS}, x}^{2}+A_{\mathrm{QRS}, y}^{2}+A_{\mathrm{QRS}, z}^{2}\right)^{1 / 2}$

The T-wave area, measured from the J-point to the end of the T wave, was calculated concordantly:

$A_{\mathrm{T} \text {-wave }}=\left(A_{\mathrm{T} \text {-wave }, x}^{2}+A_{\mathrm{T} \text {-wave }, y}^{2}+A_{\mathrm{T} \text {-wave }, z}^{2}\right)^{1 / 2}$

The spatial mean QRS-T angle reflects the spatial angle between depolarization and repolarization and was calculated as [18]:

$\alpha_{\mathrm{QRS}-T}=\operatorname{acos}\left(\frac{A_{\mathrm{QRS}, x} \times A_{\mathrm{T}-\text { wave }, x}+A_{\mathrm{QRS}, y} \times A_{\mathrm{T}-\text { wave }, y}+A_{\mathrm{QRS}, z} \times A_{\mathrm{T}-\text { wave }, z}}{\left(A_{\mathrm{QRS}, x}^{2}+A_{\mathrm{QRS}, y}^{2}+A_{\mathrm{QRS}, z}^{2}\right)^{1 / 2} \times\left(A_{\mathrm{T}-\text { wave }, x}^{2}+A_{T \text {-wave }, y}^{2}+A_{T-\text { wave }, z}^{2}\right)^{1 / 2}}\right) \times \frac{180}{\pi}$

The analysis of the 12-lead ECG, reconstruction of the VCG, and computation of parameters were automatically performed by custom software.

\section{Results}

\section{Study population}

Patient demographics are provided in Table 2. The measured ECGs of all patients showed a negative QRS complex and positive $\mathrm{T}$ wave in lead V1, absent q waves in leads I, V5 and V6, and discordant $\mathrm{T}$ waves in most of the leads.

\section{Computer simulations}

Complete simulation sets as described in the methods were only performed in patients 1 and 3 . Shifting the heart $6 \mathrm{~cm}$ to the left was not possible for patients 2,4 , and 5 due to chest boundaries. The maximum left shift for these patients was 3, 5, and $4 \mathrm{~cm}$, respectively. Fig. 2 shows the measured and simulated ECGs of the patients with their accompanying heart-torso anatomies.

Table 1

Definitions of complete LBBB according to ESC [2], the AHA [3], and Strauss [4].

\begin{tabular}{llll}
\hline & ESC & AHA & Strauss \\
\hline QRS duration & $\geq 120 \mathrm{~ms}$ & $\geq 120 \mathrm{~ms}$ & 9 $\geq 130 \mathrm{~ms}, \sigma^{\top} \geq 140 \mathrm{~ms}$ \\
QS or rS pattern & V1 with positive T-wave & - & V1-V2 \\
QS pattern & aVR with positive T-wave & - & - \\
Delayed ID-time $(\geq 60 \mathrm{~ms})$ & I and V6 & V5-V6 & - \\
Discordant T-waves & Usually & Usually & - \\
Mid-QRS notching/slurring & - & I, aVL, V5-V6 & V1-V2, V5-V6, I, aVL ( $\geq 2$ contiguous leads) \\
Absent q waves & - & I, V5-V6 & - \\
QRS axis deviation & - & May change & - \\
\hline
\end{tabular}

Abbreviations: AHA = American Heart Association, ESC = European Society of Cardiology, ID-time = intrinsicoid deflection time. 
Table 2

Patient characteristics.

\begin{tabular}{llllll}
\hline & Patient 1 & Patient 2 & Patient 3 & Patient 4 & Patient 5 \\
\hline Age (years) & 72 & 69 & 79 & 57 & 71 \\
Gender (male/female) & Female & Male & Male & Male & Male \\
Height $(\mathrm{m})$ & 1.57 & 1.82 & 1.87 & 1.60 & 1.88 \\
Weight $(\mathrm{kg})$ & 75 & 75 & 94 & 67 & 130 \\
BMI $\left(\mathrm{kg} / \mathrm{m}^{2}\right)$ & 30.4 & 22.6 & 26.9 & 26.2 & 36.8 \\
NYHA class & III-IV & II-III & II-III & II-III & III-IV \\
$\quad(\mathrm{I} / \mathrm{II} / \mathrm{III} / \mathrm{IV})$ & & & & & \\
LVEF $(\%)$ & 39 & 35 & 28 & 30 & 25 \\
\hline
\end{tabular}

Abbreviations: $\mathrm{BMI}=$ body mass index, $\mathrm{LVEF}=$ left ventricular ejection fraction, NYHA = New York Heart Association Functional classification.

\section{ECG morphology analyses}

A total of twenty-five morphology parameters were evaluated. A morphology alteration was considered present when the ECG morphology criterion was altered at least once over the entire range of a modification. The (numbered) morphology parameters and assessment are represented in Table 3.

Intrinsicoid deflection time (ID-time) $\geq 60 \mathrm{~ms}$ in leads V6 and I, notching/slurring in the precordial leads, and the RS pattern in leads V5 and V6 were altered most frequently. In contrast, QRSd, absent q waves in leads I, V5, and V6, and mainly discordant $T$ waves remained unaffected. Representative morphology alterations in the ECG signal are shown in Fig. 3.

Patients were diagnosed with ESC LBBB (LBBB $\mathrm{ESC}_{\text {) }}$ when 8 morphology parameters (parameters 1, 3 or 4, 5, 8, 9, 12, 14, and 24) were present [2]. Only patient 1 was diagnosed with $\mathrm{LBBB}_{\mathrm{ESC}}$ at baseline simulation. Throughout the geometry modifications the diagnosis of patient 1 changed four times to non- $\mathrm{LBBB}_{\mathrm{ESC}}$ mainly due to alterations in ID-time in leads I and V6 (parameters 12 and 14). Patient 2 was a non-LBBB $\mathrm{ESC}_{\mathrm{ESC}}$ at baseline, but became an $\mathrm{LBBB}_{\mathrm{ESC}}$ patient once due to the development of a QS complex and positive T wave in lead V2 (parameters 8 and 9). Patients 3, 4, and 5 were non-LBBB $\mathrm{ESC}_{\mathrm{E}}$ at baseline and remained so throughout the modifications.
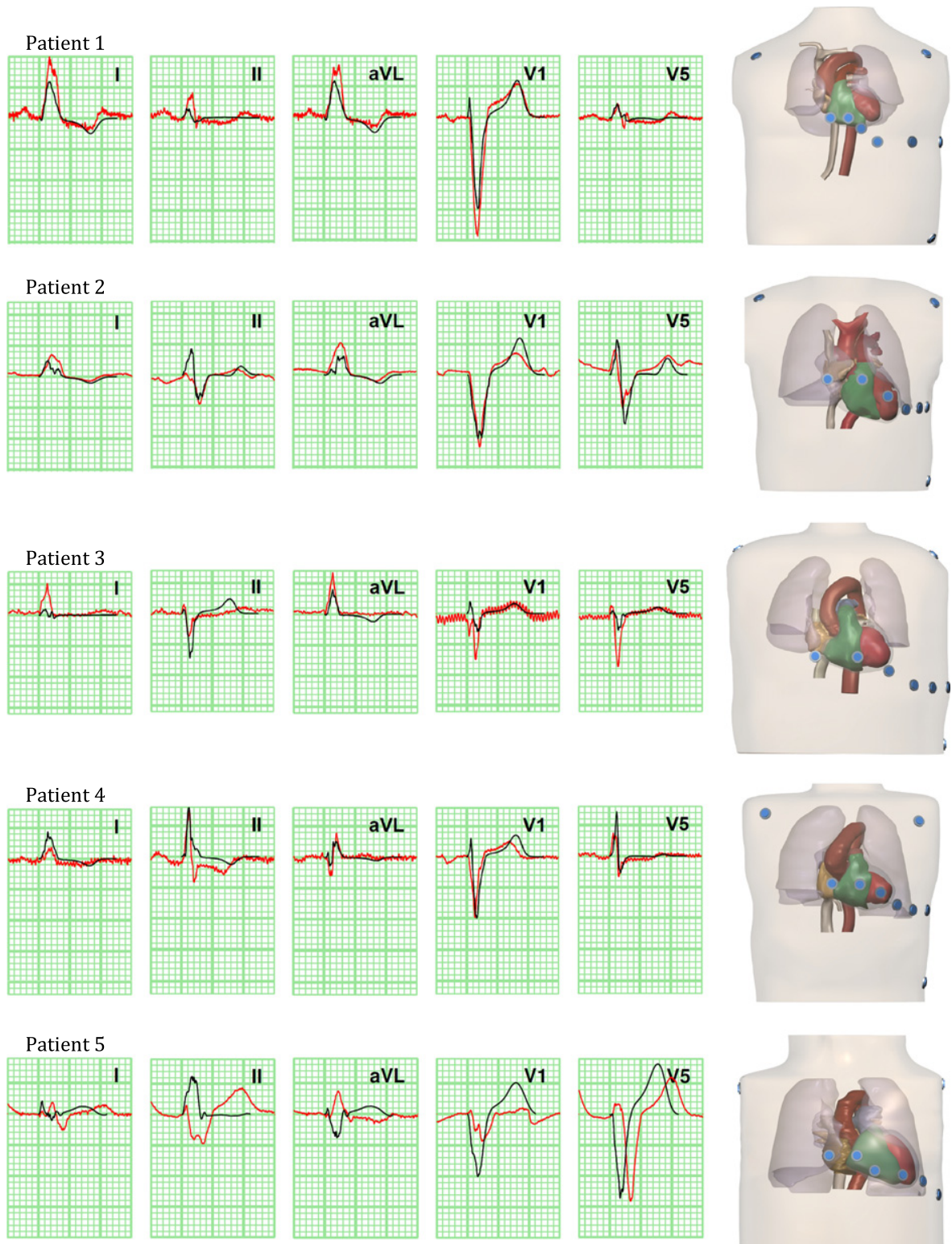
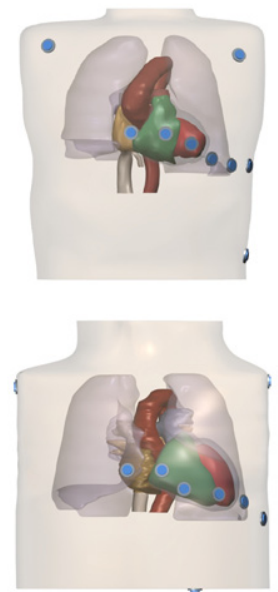
Table 3

ECG morphology alterations.

\begin{tabular}{|c|c|c|c|c|c|c|c|c|c|c|c|c|}
\hline \multicolumn{2}{|c|}{ Morphology parameters } & \multicolumn{2}{|c|}{ Patient 1} & \multicolumn{2}{|c|}{ Patient 2} & \multicolumn{2}{|c|}{ Patient 3} & \multicolumn{2}{|c|}{ Patient 4} & \multicolumn{2}{|c|}{ Patient 5} & \multirow{2}{*}{$\begin{array}{l}\text { Total no. } \\
\text { of changes }\end{array}$} \\
\hline & & BS & U-D-R-L-H-V-E & BS & U-D-R-L-H-V-E & BS & U-D-R-L-H-V-E & BS & U-D-R-L-H-V-E & BS & U-D-R-L-H-V-E & \\
\hline 1 & $\mathrm{QRSd} \geq 120 \mathrm{~ms}$ & Yes & $0-0-0-0-0-0-0$ & Yes & $0-0-0-0-0-0-0$ & No & $0-0-0-0-0-0-0$ & Yes & $0-0-0-0-0-0-0$ & Yes & $0-0-0-0-0-0-0$ & 0 \\
\hline 2 & QRSd 우 $\geq 130 \mathrm{~ms} \sigma^{\top} \geq 140 \mathrm{~ms}$ & Yes & $0-0-0-0-0-0-0$ & Yes & $0-0-0-0-0-0-0$ & No & $0-0-0-0-0-0-0$ & No & $0-0-0-0-0-0-0$ & Yes & $0-0-0-0-0-0-0$ & 0 \\
\hline 3 & V1: QS pattern & No & $0-1-0-0-0-0-0$ & Yes & $0-0-0-0-0-0-0$ & No & $0-1-0-0-0-0-0$ & & $0-0-0-0-0-0-0$ & Yes & $0-0-0-0-0-0-0$ & 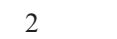 \\
\hline 4 & $\mathrm{~V} 1: \mathrm{rS}$ pattern & Yes & $0-1-0-0-0-0-0$ & No & $0-0-0-0-0-0-0$ & Yes & $0-1-0-1-0-0-0$ & Yes & $0-0-0-0-0-0-0$ & No & $0-0-0-0-0-0-0$ & J \\
\hline 5 & V1: positive $\mathrm{T}$ wave & Yes & $0-0-0-0-0-0-0$ & Yes & $0-0-0-0-0-0-0$ & Yes & $0-0-0-0-0-0-0$ & Yes & $0-0-0-0-0-0-0$ & Yes & $0-0-0-0-0-0-0$ & 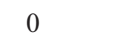 \\
\hline 6 & V2: QS pattern & No & $0-1-0-0-0-0-0$ & Yes & $0-0-0-0-0-0-0$ & No & $0-1-0-0-0-0-0$ & No & $0-0-0-0-0-0-0$ & Yes & $0-0-0-0-0-0-0$ & 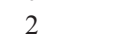 \\
\hline 7 & $\mathrm{~V} 2: \mathrm{rS}$ pattern & Yes & $0-1-0-0-0-0-0$ & No & $0-0-0-0-0-0-0$ & Yes & $0-1-0-0-0-0-0$ & Yes & $0-0-0-0-0-0-0$ & No & $0-0-0-0-0-0-0$ & 2 \\
\hline 8 & aVR: QS pattern & Yes & $0-0-0-0-0-0-0$ & No & $0-0-0-0-0-1-0$ & No & $0-0-0-0-0-0-0$ & Yes & $0-0-0-0-0-0-0$ & Yes & $0-0-0-0-0-0-0$ & 1 \\
\hline 9 & aVR: positive $T$ wave & Yes & $0-0-0-0-0-0-0$ & No & $0-0-0-0-0-1-0$ & No & $0-0-($ & Yes & $0-0-($ & Yes & $0-0-$ & 1 \\
\hline 10 & V5: RS pattern & No & $1-0-0-1-1-0-1$ & Yes & $0-0-0-0-0-0-0$ & No & $0-1-0-0-0$ & Yes & $0-0-1-0-0$ & No & $0-0-0-0-0-0-0$ & 7 \\
\hline 11 & V6: RS pattern & No & $1-0-0-0-1-0-0$ & No & $0-0-0-0-0-1-0$ & No & $0-1-0-1-0-0-0$ & No & $0-0-0-0-1-1-0$ & No & $1-1-0-1-1-1-0$ & 12 \\
\hline 12 & I: delayed ID-time $\geq$ & Yes & $0-0-1-0-1-0-0$ & & $0-0-0-0-0-0-0$ & No & $0-0-0-0-1-0-0$ & No & $0-1-0-1-0-1-0$ & No & $0-0-0-0-1-0-0$ & 7 \\
\hline 13 & V5: delayed ID-time $\geq 60 \mathrm{~ms}$ & No & $0-0-0-0-0$ & $\mathrm{~N}$ & $0-0-0-0-0-0-1$ & No & $-0-1-0$ & No & $0-0-0-0-0-0-0$ & Yes & $0-0-0-0$ & 4 \\
\hline 14 & V6: delayed ID-time $\geq 60 \mathrm{~ms}$ & Yes & $1-0-1-0-1-0-1$ & Yes & $0-0-0-0-1-0-0$ & No & $0-1-0-1-0-1-0$ & No & $0-0-0-0-0-0-0$ & Yes & $0-1-1-0-1-1-0$ & 12 \\
\hline 15 & I: absent q waves & Yes & $0-0-0-0-0-0-0$ & Yes & $0-0-0-0-0-0-0$ & Yes & $0-0-0-0-0-0-0$ & Yes & $0-0-0-0-0-0-0$ & Yes & $0-0-0-0-0-0-0$ & 0 \\
\hline 16 & V5: absent q waves & Yes & $0-0-0-0-0-0-0$ & Yes & $0-0-0-0-0-0-0$ & Yes & $0-0-0-0-0-0-0$ & Yes & $0-0-0-0-0-0-0$ & Yes & $0-0-0-0-0-0-0$ & 0 \\
\hline 17 & V6: absent q waves & Yes & $0-0-0-0-0-0-0$ & Yes & $0-0-0-0-0-0-0$ & Yes & $0-0-0-0-0-0-0$ & Yes & $0-0-0-0-0-0-0$ & Yes & $0-0-0-0-0-0-0$ & 0 \\
\hline 18 & I: mid-QRS notching/slurring & No & $0-0-0-0-0-0-0$ & Yes & $0-0-0-0-0-0-0$ & Yes & $0-0-0-0-0-0-0$ & Yes & $0-0-0-0-0-0-0$ & Yes & $0-0-0-0-0-0-0$ & 0 \\
\hline 19 & aVL: mid-QRS notching/slurring & No & $0-0-0-0-0-0-0$ & Yes & $0-0-0-0-0-0-0$ & Yes & $0-0-0-0-0-0-0$ & Yes & $0-0-0-0-0-0-0$ & Yes & $0-0-0-0-0-0-0$ & 0 \\
\hline 20 & V1: mid-QRS notching/slurring & No & $0-1-0-0-0-0-0$ & Yes & $0-1-0-0-0-0-0$ & Yes & $0-0-1-0-0-1-0$ & No & $0-1-0-0-0-0-0$ & No & $0-1-0-1-0-0-0$ & 7 \\
\hline 21 & V2: mid-QRS notching/slurring & No & $0-1-1-0-1-1-0$ & Yes & & No & & No & & No & $0-0-0-0-0-0-0$ & 8 \\
\hline 22 & V5: mid-QRS notching/slurring & Yes & $1-0-0-1-1-1-0$ & No & $1-1-1-1-0-0-0$ & Yes & $0-0-0-1-1-0-0$ & No & $0-1-0-0-0-1-0$ & Yes & $0-1-0-1-0-0-0$ & 14 \\
\hline 23 & V6: mid-QRS notching/slurring & No & $1-0-0-1-1-0-0$ & Yes & $0-0-0-1-0-0-0$ & Yes & $1-0-0-0-1-0-0$ & Yes & $0-0-0-0-0-1-0$ & Yes & $0-0-0-1-0-0-0$ & 8 \\
\hline 24 & Discordant $\mathrm{T}$ waves & Yes & $0-0-0-0-0-0-0$ & Yes & $0-0-0-0-0-0-0$ & Yes & $1-0-0-0-0-0-0$ & Yes & $0-0-0-0-0-0-0$ & Yes & $0-0-0-0-0-0-0$ & 1 \\
\hline 25 & QRS axis deviation & Yes & $0-0-0-0-0-0-0$ & No & $0-1-0-1-1-1-0$ & Yes & $0-0-0-0-0-1-0$ & No & $0-0-0-0-0-0-0$ & No & $0-0-0-0-1-0-0$ & 6 \\
\hline
\end{tabular}

Presence $(=1)$ or absence $(=0)$ of a morphology alteration at baseline simulation (BS) and after geometry modifications: shift up (U)-shift down (D)-shift right (R)-shift left (L)-rotate horizontal (H)-rotate vertical (V)-shift electrodes (E). A morphology alteration is present when the morphology parameter is changed with respect to the BS.

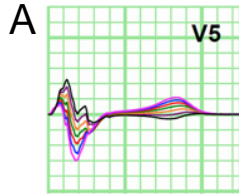

$+1 \mathrm{~cm}$ steps

B

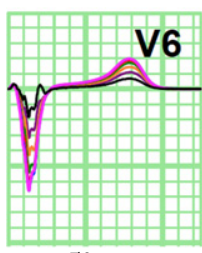

$-5^{\circ}$ steps

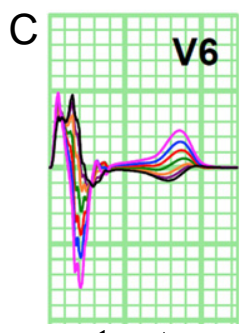

$-1 \mathrm{~cm}$ steps

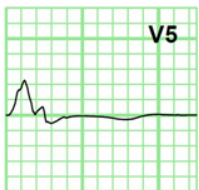

Baseline

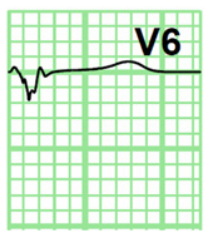

Baseline

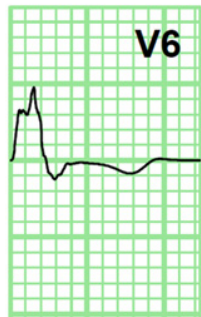

Baseline

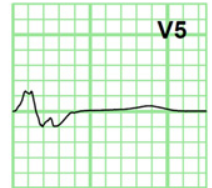

$+2 \mathrm{~cm}$

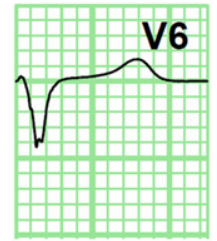

$-10^{\circ}$

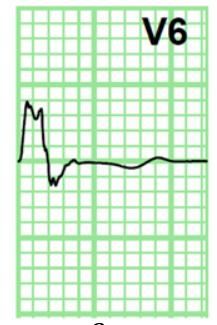

$-2 \mathrm{~cm}$

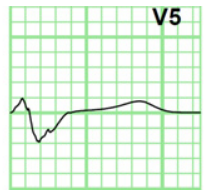

$+4 \mathrm{~cm}$

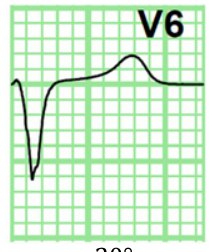

$-20^{\circ}$

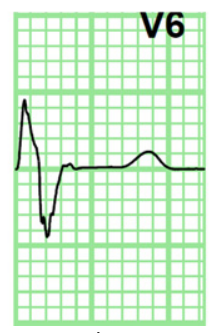

$-4 \mathrm{~cm}$

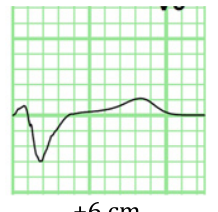

$+6 \mathrm{~cm}$

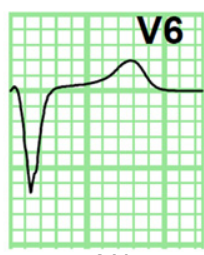

$-30^{\circ}$

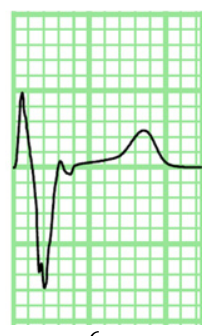

$-6 \mathrm{~cm}$

Fig. 3. Representative morphology alterations of the ECG signal due to geometry modifications. A. Patient 1 (lead V5): shifting the heart upward. The notch slowly vanishes, when the heart is shifted further upwards. B. Patient 3 (lead V6): rotating the heart to horizontal. The notch disappears and the QRS amplitude increases when the heart is positioned more horizontally. C. Patient 5 (lead V6): shifting the heart downward. A RS complex develops and the R peak and J point morphology alters, leading to varying estimations of the ID-time and QRSd. 
Table 4

Quantitative parameters at baseline and after geometry adjustments.

\begin{tabular}{|c|c|c|c|c|c|c|}
\hline & QRS duration & QRS amplitude & QRS area & QRS-T angle & T-wave amplitude & T-wave area \\
\hline \multicolumn{7}{|l|}{ Patient 1} \\
\hline Baseline simulation & $142 \mathrm{~ms}$ & $1.27 \mathrm{mV}$ & $65.3 \mathrm{mV} \cdot \mathrm{ms}$ & $175.9^{\circ}$ & $0.51 \mathrm{mV}$ & $62.6 \mathrm{mV} \cdot \mathrm{ms}$ \\
\hline Heart up-down (\%) & $99-105$ & $78-101$ & $79-103$ & $100-100$ & $78-101$ & $78-101$ \\
\hline Heart left-right (\%) & $97-104$ & $76-103$ & $74-106$ & $98-100$ & $69-103$ & $70-103$ \\
\hline Heart rotation $(\%)$ & $99-103$ & $88-101$ & $88-104$ & $100-101$ & $90-100$ & $90-100$ \\
\hline V1-V6 down (\%) & $100-100$ & $89-100$ & $88-100$ & $100-100$ & $90-100$ & $90-100$ \\
\hline \multicolumn{7}{|l|}{ Patient 2} \\
\hline Baseline simulation & $166 \mathrm{~ms}$ & $1.48 \mathrm{mV}$ & $110.1 \mathrm{mV} \cdot \mathrm{ms}$ & $177.2^{\circ}$ & $0.77 \mathrm{mV}$ & $98.9 \mathrm{mV} \cdot \mathrm{ms}$ \\
\hline Heart up-down $(\%)$ & $99-106$ & $71-110$ & $64-104$ & $98-100$ & $59-106$ & $58-106$ \\
\hline Heart left-right $(\%)$ & $98-101$ & $91-159$ & $91-141$ & $100-100$ & $89-136$ & $88-130$ \\
\hline Heart rotation $(\%)$ & $96-100$ & $87-106$ & $87-109$ & $99-100$ & $84-107$ & $83-107$ \\
\hline V1-V6 down (\%) & $100-100$ & $100-106$ & $100-104$ & $100-100$ & $100-104$ & $100-104$ \\
\hline \multicolumn{7}{|l|}{ Patient 3} \\
\hline Baseline simulation & $115 \mathrm{~ms}$ & $0.81 \mathrm{mV}$ & $29.5 \mathrm{mV} \cdot \mathrm{ms}$ & $173.5^{\circ}$ & $0.29 \mathrm{mV}$ & $34.9 \mathrm{mV} \cdot \mathrm{ms}$ \\
\hline Heart up-down $(\%)$ & $100-104$ & $66-133$ & $74-137$ & $97-101$ & $70-124$ & $70-124$ \\
\hline Heart left-right (\%) & $100-103$ & $96-125$ & $89-130$ & $100-102$ & $96-119$ & $94-118$ \\
\hline Heart rotation $(\%)$ & $97-108$ & $64-140$ & $66-142$ & $94-100$ & $69-125$ & $70-125$ \\
\hline V1-V6 down (\%) & $100-103$ & $100-111$ & $100-108$ & $100-101$ & $100-107$ & $100-106$ \\
\hline \multicolumn{7}{|l|}{ Patient 4} \\
\hline Baseline simulation & $125 \mathrm{~ms}$ & $1.01 \mathrm{mV}$ & $45.3 \mathrm{mV} \cdot \mathrm{ms}$ & $161.6^{\circ}$ & $0.33 \mathrm{mV}$ & $40.0 \mathrm{mV} \cdot \mathrm{ms}$ \\
\hline Heart up-down $(\%)$ & $99-102$ & $79-104$ & $81-116$ & $98-103$ & $75-106$ & $77-105$ \\
\hline Heart left-right (\%) & $99-101$ & $99-134$ & $100-122$ & $100-102$ & $100-119$ & $100-119$ \\
\hline Heart rotation $(\%)$ & $99-102$ & $92-115$ & $77-127$ & $99-103$ & $89-121$ & $90-121$ \\
\hline V1-V6 down (\%) & $100-100$ & $93-100$ & $88-100$ & $98-100$ & $88-100$ & $89-100$ \\
\hline \multicolumn{7}{|l|}{ Patient 5} \\
\hline Baseline simulation & $143 \mathrm{~ms}$ & $1.53 \mathrm{mV}$ & $103.6 \mathrm{mV} \cdot \mathrm{ms}$ & $173.9^{\circ}$ & $0.67 \mathrm{mV}$ & $85.5 \mathrm{mV} \cdot \mathrm{ms}$ \\
\hline Heart up-down $(\%)$ & $94-110$ & $64-131$ & $63-135$ & $98-101$ & $61-136$ & $62-133$ \\
\hline Heart left-right (\%) & $97-110$ & $94-145$ & $100-118$ & $100-100$ & $99-118$ & $100-115$ \\
\hline Heart rotation $(\%)$ & $98-106$ & $83-109$ & $84-115$ & $100-100$ & $81-114$ & $82-115$ \\
\hline V1-V6 down (\%) & $100-101$ & $84-100$ & $84-100$ & $100-100$ & $83-100$ & $83-100$ \\
\hline
\end{tabular}

Patients were diagnosed with AHA LBBB (LBBB $\left.{ }_{\mathrm{AHA}}\right)$ when 11 morphology parameters (parameters 1, 13-19, 2224) were present [3]. Only patient 5 was diagnosed with $\mathrm{LBBB}_{\mathrm{AHA}}$ at baseline. Throughout the geometry modifications patient 5 changed five times to a non- $\mathrm{LBBB}_{\mathrm{AHA}}$ as a consequence of ID-time alterations in lead V6 (parameter 14) and notching/slurring changes in leads V5 and V6 (parameters 22-23). Patients 1-4 were non-LBBB $\mathrm{AHA}_{\mathrm{A} A}$ at baseline and maintained their diagnosis throughout the geometry modifications.

Patients were diagnosed with Strauss LBBB $\left(\mathrm{LBBB}_{\text {Strauss }}\right)$ when 5 parameters were present (parameters 2,3 or 4,6 or 7 , at least 2 contiguous leads from parameters 18-23) [4]. At baseline patients 2 and 5 were diagnosed with $\mathrm{LBBB}_{\text {Strauss }}$ and this diagnosis was maintained throughout the modifications. Patients 1,3 , and 4 were diagnosed with non-LBBB ${ }_{\text {Strauss }}$ at baseline. Patients 3 and 4 maintained the non-LBBB ${ }_{\text {Strauss }}$ diagnosis throughout the modifications, while patient 1 changed to an $\mathrm{LBBB}_{\text {Strauss }}$ once due to the emergence of a notch (parameters 20 and 21). The LBBB/non-LBBB diagnoses evaluation is provided in Supplementary Table 1.

\section{VCG quantitative analyses}

All quantitative parameter values at baseline and ranges after geometry modifications are provided in Table 4 . Ranges are expressed as percentages of the baseline value. Absolute ranges are provided in Supplementary Table 2.
Alterations in QRSd were generally small, except for patient 5 when the heart was shifted up and down. QRS-T angle also remained practically unaffected by the geometry modifications.

Geometry modifications resulted in relatively large QRS amplitude and $\mathrm{T}$-wave amplitude alterations (several dozens of percents in some cases). These large alterations were also present in QRS area and T-wave area, as could be expected since these parameters are functions of amplitudes and durations. Alterations in QRS amplitude and QRS area were more prominent in patient 3 when the heart was rotated. Alterations in $\mathrm{T}$-wave amplitude and $\mathrm{T}$-wave area were greatest in patient 5 when the heart was shifted up and down.

Shifting the heart to the right led to a decrease of voltage-dependent parameters in patients 1,2 , and 5 , but not in patients 3 and 4 . This may be explained by the position of the ventricles in relation to the precordial electrodes. When the heart was shifted to the right in patients 1,2 , and 5 , the distance between the precordial electrodes and the LV increased, while in patients 3 and 4 this distance initially increased but eventually decreased when the LV was close to V1 and V2.

For all parameters, shifting the heart along the z-axis resulted in the largest parameter alterations. Alterations in QRSd, QRS area, QRS-T angle, and T-wave area are represented in Fig. 4.

\section{Discussion}

The influence of geometrical factors on ECG parameters has been extensively investigated in the past $[5-7,19]$, but 

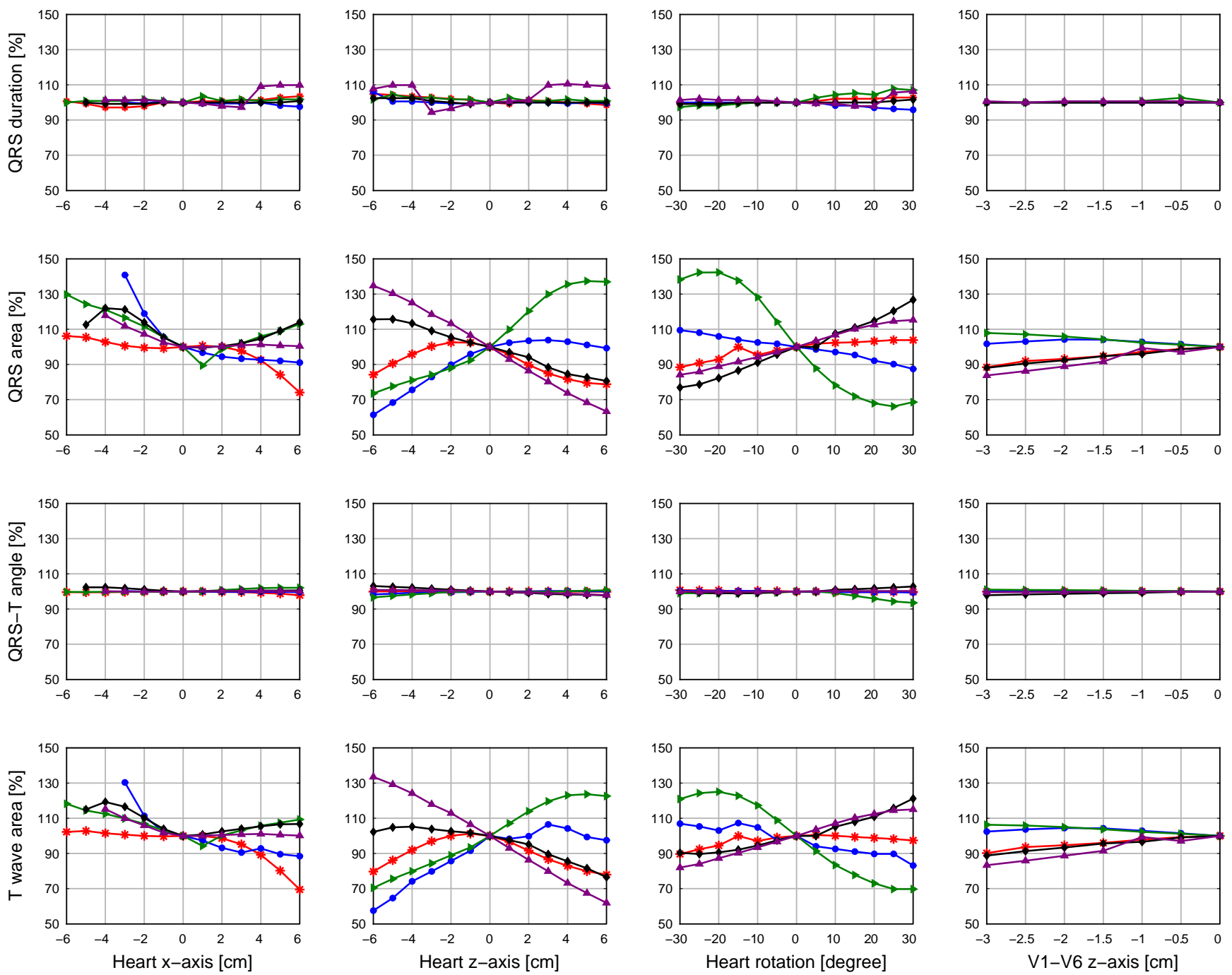

Fig. 4. Relative parameter values (y-axis) as a function of geometry modifications ( $\mathrm{x}$-axis) as described previously in Fig. 1. Colored lines represent patient 1 (red), patient 2 (blue), patient 3 (green), patient 4 (black), and patient 5 (purple). Note that throughout the geometry modifications, the QRSd and QRS-T angle remain relatively constant, while the QRS area and T-wave area are severely affected.

the influence of geometry on QRS morphology and VCG parameters has not been studied before in patients with wide QRS complexes. Our results, based on an in-silico approach using patient-specific geometries, demonstrate that morphological features of the ECG (in particular notching/slurring and ID-time) and voltage-dependent VCG parameters (QRS amplitude, QRS area, T-wave amplitude, and T-wave area) are severely affected by geometry modifications, influencing the diagnosis of LBBB.

The presence of notching/slurring as a criterion for LBBB was proposed by Strauss et al. and has been incorporated in the AHA and ESC guidelines [3,4]. Typical notching in the presence of LBBB starts when the depolarization wavefront breaks through the LV endocardium and ends when the epicardium of the lateral wall is reached [4]. In our study, notching/slurring patterns in the precordial leads V1, V2, V5, and V6 were affected by geometrical factors, but were remarkably unaffected in the frontal leads I and aVL. Body surface mapping studies in LBBB patients have demonstrated strong potential gradients near the precordial electrodes $[20,21]$. It is likely that when the position or orientation of the heart is modified or the electrodes are shifted, this gives rise to relatively large changes in the measured ECG.

ID-time changes in our results were present in leads I, V5, and V6, often in the presence of multiple peaks in the R wave due to notching. Throughout the geometry modifications the maximum amplitude in the $\mathrm{R}$ wave switched between the multiple peaks, leading to jumps in estimated ID time. We therefore assume that the ID-time alterations may partly occur due to notch morphology modifications (Fig. 3).

The LBBB/non-LBBB diagnosis changed in 2 patients according to the ESC criteria, in 1 patient according to the AHA criteria, and in 1 patient according to the Strauss criteria. This particularly occurred as a consequence of notching/slurring and ID-time alterations. Most of our patients were non-LBBB at baseline due to lack of multiple LBBB parameters. Modifying the geometry led to a few morphology parameters changes, insufficient to overcome this. However, we observed that in patients with LBBB at baseline, diagnosis alterations frequently occurred as a result of a single parameter alteration. 
In our study geometrical factors altered the parameters QRS amplitude, QRS area, T-wave amplitude, and T-wave area severely, while QRSd and QRS-T angle remained relatively unaffected.

The maximum decrease and increase of QRSd were small enough not to traverse the QRSd thresholds of 120,130, and $140 \mathrm{~ms}$. Greatest changes in QRSd were observed in patient 5 after shifting the heart along the z-axis. Upon careful evaluation of the vector magnitude of the VCG of this patient we found multiple negative deflections around the end of the QRS complex. Morphology alterations of this notch due to geometry modifications led to differences in the local minimum of the vector magnitude which our software uses to calculate the QRSd.

The ECG of patient 5 was particularly sensitive to anatomical changes, especially in the precordial leads. We think that this is due to a relatively large heart size in combination with a relatively small torso. A short distance between the heart and the precordial electrodes leads to a relatively large contribution from nearby myocardium, which is more sensitive to shift than the more remote contributions.

The observation that QRS area may change by dozens of percents due to alterations in heart position is relevant since Van Deursen et al. [15] demonstrated that a cutoff value of $98 \mu \mathrm{V}$-s identified CRT responders with an odds ratio (OR) of 10.2. Similarly, the OR of T-wave area to predict CRT response is 1.172 per $10 \mu \mathrm{V}$-s [16]. Our observed parameter value alterations may lead to a change in prediction of CRT response based on QRS area in patients 2 and 5 and based on T-wave area in patients 3 and 4 .

For all parameters, shifting the precordial electrodes downwards resulted in alterations similar to shifting the heart up by the same amount. We chose to shift the electrodes up to $3 \mathrm{~cm}$, as it was found in clinical practice that the average distance from the actual electrode position to the prescribed location was $2.9 \mathrm{~cm}$ [7]. However, VCG parameter alterations after shifting the electrodes by such small amounts were minimal in our results $(-17 \%$ and $+8 \%)$. Hoekema et al. [22] attempted to reduce the interindividual variability of ECGs by placing the electrodes on the torso with reference to the heart position instead of to the ribs. Their approach failed to reduce the relative variability of the QRS complex. Our results confirm that the relative position of heart and electrodes contributes little to the inter-individual variability.

\section{Future prospects and clinical implications}

Precise diagnosis of IVCDs may be important to predict CRT outcome. In the present study we have used our models as predictive tools, assuming that the anatomical effects on the ECG are represented well enough. The same models can be used as investigative tools, as we have shown in previous work [9]. By trial and error one can find a set of model parameters that allows the model to optimally match the measured signals. These model parameters describe the individual pathology in mechanistic terms and can be seen as a form of diagnosis. Because patient-tailored model anatomies are used, this diagnosis is immune to the interindividual variability that plagues criteria-based diag- nosis. However, this method is still in its infancy. The long time it takes to create the individual anatomical models, run the simulations, and analyze the results, as well as the lack of validation of the outcomes, does not allow this method to be used clinically yet.

\section{Limitations}

There are several limitations in this study that need to be addressed.

Firstly, matching the baseline simulation with the measured ECG required extensive tuning and numerous test simulations. A perfect and unique representation of the true ECG was not always reached. For our study, which aimed at investigating how geometrical factors affect ECG parameters, a correct representation of the underlying electrophysiology was not crucial.

Secondly, the present study was performed on only 5 patients based on individual patient-tailored models and therefore aims at providing additional insight in the basic mechanisms of the ECG rather than providing statistical statements. However, the strength of a simulation study compared to experimental or clinical studies is its ability to keep all but one source of variation unaffected. In addition, by including patients with a wide range in QRS duration and morphology we covered a wide range of baseline situations.

Thirdly, the magnitude of the maximum shifts applied $(6 \mathrm{~cm})$ is large, especially when applied in small and slender patients. However the physical constraints of each patient's heart-torso anatomy were taken into account when these shifts were applied.

Lastly, we used the Kors transformation to calculate the VCG from the 12-lead ECG. We chose to do so because true VCG electrodes are rarely used in recent literature. Among all methods to estimate a VCG from a 12-lead ECG, the Kors matrix is the most accurate $[23,24]$.

\section{Conclusion}

Our results demonstrate that geometrical factors determine the presence of notching/slurring, RS patterns, and ID-times on the ECG and the magnitude of voltagedependent parameters on the VCG. This indicates that the heart-torso geometry with respect to the electrode positions must be considered for accurate diagnosis of IVCDs.

\section{Acknowledgments}

This work was supported by a grant from the Swiss National Supercomputing Centre (CSCS) under project ID 397. The authors gratefully acknowledge financial support by Fondazione Cardiocentro Ticino, the Theo Rossi di Montelera Foundation, the Mantegazza Foundation, and FIDINAM to the Center of Computational Medicine in Cardiology.

\section{Appendix A. Supplementary data}

Supplementary data to this article can be found online at http://dx.doi.org/10.1016/j.jelectrocard.2015.05.004. 


\section{References}

[1] Auricchio A, Prinzen FW. Non-responders to cardiac resynchronization therapy: the magnitude of the problem and the issues. Circ J 2011;75:521-7.

[2] Bayés de Luna A, Batchvarov VN, Malik M. Chapter 1: the morphology of the electrocardiogram. In: Camm AJ, editor. The ESC textbook of cardiovascular medicine. Blackwell Publishing; 2006.

[3] Surawicz B, Childers R, Deal BJ, Gettes LS, Bailey JJ, Gorgels A, et al. AHA/ACCF/HRS recommendations for the standardization and interpretation of the electrocardiogram: part III: intraventricular conduction disturbances: a scientific statement from the American Heart Association Electrocardiography and Arrhythmias Committee, Council on Clinical Cardiology; the American College of Cardiology Foundation; and the Heart Rhythm Society. Endorsed by the International Society for Computerized Electrocardiology. J Am Coll Cardiol 2009;53:976-81.

[4] Strauss DG, Selvester RH, Wagner GS. Defining left bundle branch block in the era of cardiac resynchronization therapy. Am J Cardiol 2011;107:927-34.

[5] MacLeod RS, Ni Q, Punske B, Ershler PR, Yilmaz B, Taccardi B. Effects of heart position on the body-surface electrocardiogram. J Electrocardiol 2000;33:229-37.

[6] Hoekema R, Uijen GJ, van Oosterom A. Geometrical aspects of the interindividual variability of multilead ECG recordings. IEEE Trans Biomed Eng 2001;48:551-9.

[7] Schijvenaars BJ, van Herpen G, Kors JA. Intraindividual variability in electrocardiograms. J Electrocardiol 2008;41:190-6.

[8] Brignole M, Auricchio A, Baron-Esquivias G, Bordachar P, Boriani G, Breithardt OA, et al. 2013 ESC guidelines on cardiac pacing and cardiac resynchronization therapy: the Task Force on cardiac pacing and resynchronization therapy of the European Society of Cardiology (ESC). Developed in collaboration with the European Heart Rhythm Association (EHRA). Eur Heart J 2013;34:2281-329.

[9] Potse M, Krause D, Kroon W, Murzilli R, Muzzarelli S, Regoli F, et al. Patient-specific modeling of cardiac electrophysiology in heart-failure patients. Europace 2014;16:iv56-61.

[10] Potse M, Dubé B, Richer J, Vinet A, Gulrajani RM. A comparison of monodomain and bidomain reaction-diffusion models for action potential propagation in the human heart. IEEE Trans Biomed Eng 2006;53:2425-35.

[11] Bacharova L, Mateasik A, Krause R, Prinzen FW, Auricchio A, Potse $M$. The effect of reduced intercellular coupling on electrocardiographic signs of left ventricular hypertrophy. J Electrocardiol 2011;44:571-6.
[12] Corlan AD, Macleod RS, De Ambroggi L. The effect of intrathoracic heart position on electrocardiogram autocorrelation maps. J Electrocardiol 2005;38:87-94.

[13] Wenger W, Kligfield P. Variability of precordial electrode placement during routine electrocardiography. J Electrocardiol 1996;29:179-84.

[14] Borleffs CJ, Scherptong RW, Man SC, van Welsenes GH, Bax JJ, van Erven L, et al. Predicting ventricular arrhythmias in patients with ischemic heart disease: clinical application of the ECG-derived QRS-T angle. Circ Arrhythm Electrophysiol 2009;2:548-54.

[15] van Deursen CJ, Vernooy K, Dudink E, Bergfeldt L, Crijns HJ, Prinzen FW, Wecke L. Vectorcardiographic QRS area as a novel predictor of response to cardiac resynchronization therapy. J Electrocardiol 2015;48:45-52.

[16] Engels EB, Vegh EM, van Deursen CJ, Vernooy K, Singh JP, Prinzen FW. T-wave area predicts response to cardiac resynchronization therapy in patients with left bundle branch block. J Cardiovasc Electrophysiol 2015;26:176-83.

[17] Kardys I, Kors JA, van der Meer IM, Hofman A, van der Kuip DA, Witteman JC. Spatial QRS-T angle predicts cardiac death in a general population. Eur Heart J 2003;24:1357-64.

[18] Oehler A, Feldman T, Henrikson CA, Tereshchenko LG. QRS-T angle: a review. Ann Noninvasive Electrocardiol 2014;19:534-42.

[19] Fowler NO, Braunstein JR. Anatomic and electrocardiographic position of the heart. Circulation 1951;3:906-10.

[20] Musso E, Stilli D, Macchi E, Regoliosi G, Brambilla C, Francescon P, et al. Body surface maps in left bundle branch block uncomplicated or complicated by myocardial infarction, left ventricular hypertrophy or myocardial ischemia. J Electrocardiol 1987;20:1-20.

[21] Sohi GS, Flowers NC, Horan LG, Sridharan MR, Johnson JC. Comparison of total body surface map depolarization patterns of left bundle branch block and normal axis with left bundle branch block and left-axis deviation. Circulation 1983;67:660-4.

[22] Hoekema R, Uijen GJH, van Erning L, van Oosterom A. Interindividual variability of multilead electrocardiographic recordings influence of heart position. J Electrocardiol 1999;32:137-48.

[23] Cortez DL, Schlegel TT. When deriving the spatial QRS-T angle from the 12-lead electrocardiogram, which transform is more Frank: regression or inverse Dower? J Electrocardiol 2010;43:302-9.

[24] Kors JA, van Herpen G, Sittig AC, van Bemmel JH. Reconstruction of the Frank vectorcardiogram from standard electrocardiographic leads: diagnostic comparison of different methods. Eur Heart J 1990;11:1083-92. 